\title{
EFFECT OF FISH-OIL-ENRICHED INTRAVENOUS LIPID EMULSION ON SYSTEMIC INFLAMMATORY RESPONSE SYNDROME IN POST INTESTINAL SURGERY CHILDREN
}

\author{
Khadijah Rizky Sumitro ${ }^{1 *}$, Meta Herdiana Hanindita ${ }^{2}$, Nur Aisiyah Widjaja ${ }^{3}$, Roedi Irawan ${ }^{4}$ \\ ${ }^{1-4}$ Department of Child Health, Faculty of Medicine, Universitas Airlangga, Indonesia \\ ${ }^{1-4}$ Dr Soetomo General Hospital, Surabaya, Indonesia \\ E-mail:khadijahrizkysumitro@gmail.com
}

\begin{abstract}
Intravenous lipid emulsions (IVLE) is one of the compositions of the parenteral nutrition (PN) that recommended in children who cannot meet their caloric requirements especially after small intestinal surgery and differentiated by their inflammatory effects. The first generation IVLE is proinflammatory, the second and the third are inflammatory neutral, while the fourth (fish-oil-enriched) is anti-inflammatory. The objective of this study is to investigate the effect of fishoil enriched IVLE on systemic inflammatory response syndrome (SIRS) in children after small intestinal surgery. A retrospective study using medical record was undertaken in children after small intestinal surgery admitted Dr. Soetomo Hospital, Surabaya in 2016-2017. Children with duodenal and jejunoileal atresia who had PN for at least three days were included. The types of IVLE used are FOLE and non-FOLE. Definition of SIRS was based on International Pediatric Sepsis Consensus Conference in 2005. A total of 25 children were included in this study. There were $44 \%$ children received FOLE and 48\% received non-FOLE while $8 \%$ children received no IVLE. Median duration and dose of IVLE was $8(5-15,5)$ days and 1,8 (1,25-2) g/kg/day. SIRS was significantly more common in girls (OR $995 \% \mathrm{CI}$ 1,3-63,0; $\mathrm{p}=0,036)$ and in the non-FOLE children (OR 8,0 95\% CI 1,24 - 51,50; $\mathrm{p}=0,022$ ).
\end{abstract}

Keywords: children, intravenous lipid emulsion, fish oil, SIRS, small intestinal surgery

\section{INTRODUCTION}

Parenteral Nutrition (PN) is indicated for infants and children who cannot meet nutrient need through oral and enteral in (American Society for Parenteral and Enteral Nutrition (ASPEN), 2002). The European Society for Clinical Nutrition and Metabolism (ESPEN) and The Canadian Society of Clinical Neurophysiologist (CSCN) clinical guidelines that recommend enteral nutrition that can be given within 24-48 hours after the patient gets treatment, specifically in patients who treated in the ICU/PICU (Singer et al., 2009). This is similar to the recommendation of the European Community to Pediatric Gastroenterology of Hepatology and Nutrition (ESPGHAN), ie parenteral nutrition must be given immediately if nutritional intake cannot be fulfilled orally or enterally (Koletzko et al., 2008; Koletzko et al., 2005). Parenteral nutrition (PN) is one of the post-operative management therapies that must be given within 24 to 48 hours after surgery (Wesson, 2018).
Intravenous fat emulsion (IVLE) is one component in parenteral nutrition that plays an important role as an energy source and source of essential fatty acids in preventing essential fatty acid deficiency (Biesboer and Stoehr, 2016; Herman et al., 2011; Wu et al., 2014). ESPGHAN and ESPEN believe that essential fatty acids should be given to patients who receive parenteral nutrition (Van Goudoever and Vlaardingerbroek, 2013).

The American Society for Parenteral and Enteral Nutrition / ASPEN (2012) classifies the generation of intravenous fat emulsions into 4 categories based on fatty acid derivatives and the inflammatory response generated by the intravenous fat formula itself (Vanekel et al., 2012). The first generation containing $100 \%$ soybean oil (SO) is a pro-inflammatory, the second generation (50:50 mixture of SO and mediumchain triglycerides / MCT) and third one (80:20 olive oil / OO and SO) is neutral inflammation while the fourth generation accompanied by fish 
oil (FO) is anti-inflammatory (Biesboer and Stoehr, 2016).

Conventional or previously used intravenous fat emulsions are IVLE that is not enriched with fish oil (non-FOLE). Increased markers of inflammation and the incidence of infection as well as a longer hospital stay were reported to be higher in patients who received non-FOLE during hospitalization. This type of fat emulsion also has limitations to be use in children with a history of previous soy allergies (Biesboer and Stoehr, 2016; Calder et al., 2010). Previous studies also reported that soybean oil can disrupt the flow of bile so that it can cause deposits to the gallstones (Biesboer and Stoehr, 2016).

Various studies conducted in adult patients show a lower incidence of infection in patients who get IVLE fortified fish fat (FOLE) (Klek, 2016). Until now, information about the effects of fish oil-enriched IVLE on the inflammatory response, especially in children after gastrointestinal surgery is very limited and no studies have been done at Dr. Soetomo, General Hospital Surabaya before.

This study aims to determine the differences in systemic inflammatory response (Systemic Inflammatory Response Syndrome / SIRS) in postgastrointestinal surgery pediatric patients who get IVLE enriched fish oil (FOLE) and not-enriched fish oil (non-FOLE).

\section{METHODS}

This study has obtained ethical clearance from the Health Research Ethics Committee Dr. Soetomo Hospital Surabaya with number 0170 / KEPK / IV / 2018 and is a retrospective study using medical records conducted in children after gastrointestinal surgery in Dr. Soetomo Hospital from January 1, 2016 to December 31, 2017 with total sample of 38 children. The sample selection used was purposive sampling. Children with duodenal and jejunoileal atresia who have had gastrointestinal surgery and received parenteral nutrition therapy for at least 3 days were inclusion criteria. The exclusion criteria were including uncomplete medical record data that will not support the research. From a total of 38 children with duodenal and jejunoileal atresia who underwent gastrointestinal surgery, 25 children met the inclusion and exclusion criteria.
The definition of Systemic Inflammatory Response Syndrome (SIRS) was based on the 2005 International Pediatric Sepsis Consensus Conference. SIRS was diagnosed if there was $\geq 2$ out of 4 of the following criteria are found: temperature abnormalities $\left(>38.5^{\circ} \mathrm{C}\right.$ or $\left.<36^{\circ} \mathrm{C}\right)$, tachycardia (heart rate $>180$ beats/minute) or bradycardia (heart rate $<100$ beats/minute, especially for ages $<1$ year), tachypnea (respiratory rate $>50$ beats/minute for ages $0-7$ days and $>40$ times/minute for ages $7-28$ days), leukocytosis (leukocyte count $>34 \times 10^{3} / \mathrm{mm}^{3}$ for ages $0-7$ days and $>19.5 \times 10^{3} / \mathrm{mm}^{3}$ for ages $7-28$ days) or leukopenia (leukocyte count $<5 \times 10^{3} / \mathrm{mm}^{3}$ ). Temperature and leukocyte abnormalities must be fulfilled by one of the criteria (Goldstein et al., 2005). The type of IVLE data used was obtained from medical records.

The main variables in this study were SIRS and IVLE type classified into a categorical scale so as to assess the outcome differences of children who get FOLE and non-FOLE were analyzed using the Chi Square test with a $\alpha<0.05$ as significant value. Data was displayed in the median (interquartile range) because it is not normally distributed.

\section{RESULTS AND DISCUSSIONS}

Thirteen children $(52 \%)$ had jejunoileal obstructions. Fourteen children $(56 \%)$ were male children. The median duration of parenteral nutrition use is $13(9.5-20.5)$ days with the median length of use and intravenous fat emulsion dose for 8 (5-15.5) days and $1.8(1.25-2)$ grams $/ \mathrm{kg} /$ day. The basic characteristics of the research subjects are shown in table 1 .

Based on the results of this study, 12 out of 23 children were met SIRS criteria and SIRS was more common in children who received intravenous fat emulsions that were not enriched with fish oil (non-FOLE) compared to children who received intravenous fat emulsions enriched with fish oil (FOLE) (OR $=8.0 ; 95 \%$ CI $1.24-51.5 ; \mathrm{p}$ $=0.022$ ) (Table 2). This systemic inflammatory response syndrome was also found in two children who did not get IVLE during treatment. The risk of SIRS increased 9-fold in girls compared to boys (OR 9 95\% CI 1.3-63.0; $\mathrm{p}=0.036$ ). 
Table 1. Basic Characteristics of Subjects

\begin{tabular}{lccc}
\hline \multicolumn{1}{c}{ Characteristic } & $\begin{array}{c}\text { Total } \\
\text { n (\%) }\end{array}$ & Min & Max \\
\hline Sex & $14(56.0)$ & & \\
• Boys & $11(44.0)$ & & \\
- Girls & $9(8.0)$ & 0 & 18 \\
Age while hospitalized (day)* & $19(24.0)$ & 3 & 42 \\
Length of hospitalization (day)* & $14(11.0)$ & 3 & 41 \\
Length of TPN (day)* & $8(10.5)$ & 0 & 39 \\
Length of IVLE (day)* & & & \\
Type of IVLE & $12(48.0)$ & & \\
• Non-FOLE & $11(44.0)$ & & \\
• FOLE & $2(8.0)$ & & \\
IVLE dosage (gram/kg/day)* & $1,8(0.75)$ & 0 & 2,7 \\
SIRS & $14(56.0)$ & & \\
\hline
\end{tabular}

*median (IQR)

Research on the effects of IVLE in children after gastrointestinal surgery is very limited. A similar study conducted by Almossawi et al retrospectively on 50 critically ill and postoperative children showed different results from this study. The group of children who got first, second and fourth generation IVLE did not have a difference in the number of sepsis ( $5 / 8$ vs $11 / 12$ vs $15 / 27 ; p=0.08$ ) (Almossawi et al., 2013).

Research on the effects of IVLE is more common in adult patients. Meta-analysis conducted by Chen et al. in 892 adult patients after major gastrointestinal surgery found that the group with IVLE enriched with fish oil had a significantly lower incidence of post-operative infections (OR: $0.56 ; 95 \%$ CI 0.32 - 0.98; $\mathrm{p}=0,04 ; \mathrm{I}^{2}=0 \%$ ) (Chen et al., 2010). Li et al. also conduct a meta-analysis of 1487 adult patients after major gastrointestinal surgery and found that IVLE enriched fish oil significantly reduced infection rates (OR: 0.53 ; $95 \%$ CI $0.35-0.81 ; \mathrm{p}=0.003 ; \mathrm{I}^{2}=0 \%$ ) (Li et al., 2013). The results of a recent meta-analysis conducted by Manzanares et al. in 733 critically ill adult patients found that patients with IVLE enriched with fish oil significantly reduced the risk of infection $(\mathrm{RR}=0.65 ; 95 \%$ CI $0.44-0.94 ; \mathrm{p}=$ $0.02 ; \mathrm{I}^{2}=0 \%$ ) (Manzanares, 2015).

Inflammation is the body's normal defense response to infections or other stimuli such as trauma, surgery and others characterized by redness, swelling, heat, pain and impaired function (Calder, 2012). The inflammatory mechanism caused by the first generation IVLE is caused by the fatty acid derivatives contained therein, namely long chain triglycerides (LCT) and $\omega-6$ essential fatty acids (EFA). LCT interferes the role of phagocytosis and chemotaxis in the immune system which can increase the risk of infection (Vanek et al., 2012). Essential fatty acids $\omega-6$ which are precursors for the formation of prostaglandin $\mathrm{E}_{2}\left(\mathrm{PGE}_{2}\right)$, prostaglandin $\mathrm{I}_{2}$ $\left(\mathrm{PGI}_{2}\right)$ and thromboxane $\mathrm{A}_{2}$ (TXA2) through the cyclooxygenase (COX) pathway and the formation of leukotriene $\mathrm{B}_{4}\left(\mathrm{LTB}_{4}\right)$, leukotriene $\mathrm{C}_{4}$ $\left(\mathrm{LBC}_{4}\right)$ and thromboxane $\mathrm{A} 2$ (TXA2) through the cyclooxygenase (COX) pathway and the formation of leukotriene B4 (LTB4), leukotriene C4 (LBC4) and thromboxane A2 (TXA2) LTE4) through the lipoxygenase (LOX) pathway from arachidonic acid (AA) acts as a pro-inflammatory through interleukin-6 (IL-6) (Figure 1).

Fish oil (FO) contained in fourth generation IVLE contains omega-3 essential fatty acids which are precursors for the formation of prostaglandin $E_{3}$ $\left(\mathrm{PGE}_{3}\right)$, prostaglandin $\mathrm{I}_{3}\left(\mathrm{PGI}_{3}\right)$ and thromboxane $\mathrm{A}_{3}\left(\mathrm{TXA}_{3}\right)$ through the cyclooxygenase (COX) pathway and the formation of leukotriene $\mathrm{B}_{5}$ $\left(\mathrm{LTB}_{5}\right)$, leukotriene $\mathrm{C}_{5}\left(\mathrm{LBC}_{5}\right)$ and leukotriene $\mathrm{E}_{5}$ $\left(\mathrm{LTE}_{5}\right)$ through the lipoxygenase (LOX) pathway from eikosapentanoid acid (EPA) (Figure 1) (Chang et al., 2012; Herman et al., 2011; Vanek et al., 2012). Omega-3 directly inhibits the activity of omega-6 essential fatty acids (EFA) by substituting AA into EPA and inhibits AA production

Table 2. Relationship between Intravenous Fat Emulsion Type and Systemic Inflammatory Response Syndrome

\begin{tabular}{|c|c|c|c|c|c|c|c|}
\hline & & \multicolumn{2}{|c|}{ Type of Intravenous Fat Emulsion } & \multirow[b]{2}{*}{ Total } & \multirow[b]{2}{*}{$p$} & \multirow[b]{2}{*}{$O R$} & \multirow[b]{2}{*}{$95 \% C I$} \\
\hline & & $\begin{array}{c}\text { non-FOLE } \\
\text { n (\%) }\end{array}$ & $\begin{array}{l}\text { FOLE } \\
\text { n (\%) }\end{array}$ & & & & \\
\hline \multirow{2}{*}{ SIRS } & Yes & $9(75.0)$ & $3(27.3)$ & 12 & & & \\
\hline & No & $3(25.0)$ & $8(72.7)$ & 11 & 0.022 & 8.0 & $1.24-51.50$ \\
\hline \multicolumn{2}{|c|}{ Total } & 12 & 11 & 23 & & & \\
\hline
\end{tabular}


and indirectly by changing the expression of inflammatory genes at the level of transcription factors and decreasing proinflammatory cytokines. The inhibited AA production causes an increase in EPA production so that the mediator shifts towards anti-inflammatory conditions (Calder, 2004; Chang et al., 2012).

This was the first retrospective study to assess systemic inflammatory response syndrome (SIRS) in children after gastrointestinal surgery who get IVLE enriched fish oil in Surabaya. One limitation of this study is that this study was collected in only one research center with a very limited number of samples in the pediatric population with gastrointestinal surgery so that the results of this study cannot be generalized to pediatric patients with other surgeries other than gastrointestinal surgery or in children without surgery.

\section{CONCLUSION}

Boys and children who get intravenous fat emulsions (IVLE) enriched with fish oil (FOLE) as a component of parenteral nutrition after gastrointestinal surgery have a lower incidence of systemic inflammatory response syndrome (SIRS). Further research is expected to be carried out with a larger number of samples at several study centers prospectively by assessing the inflammatory response of various inflammatory markers that are not only based on clinical.

\section{REFERENCES}

Almossawi, O., Meadows, N., O'Brien, S., Bierley, N., \& Wong, I. (2013). The use of SMOFLipid in critically ill and post surgical children on picu: a retrospective cohort study. Abstr. from Neonatal Paediatr. Pharm. Groups.

Biesboer, Ann N., \& Stoehr, N.A. (2016). A product review of alternative oil- based intravenous fat emulsions. Nutr. Clin. Pract. 31, 610-8. doi: $10.1177 / 0884533616661174$

Calder, P. C. (2004). n-3 Fatty acids, inflammation, and immunity - relevance to postsurgical and critically III patients. Lipids. 39(12): 1147-1161. doi: $10.1007 / \mathrm{s} 11745-004-1342-\mathrm{z}$

Calder, P. C. (2012). Fatty acids: long-chain fatty acids and inflammation. Proc. Nutr. Soc. 71, 284-9. doi: 10.1017/S0029665112000067

Calder, P. C., Jensen, G. L., Koletzko, B. V., Singer,
P., \& Wanten, G. J. A. (2010). Lipid emulsions in parenteral nutrition of intensive care patients: Current thinking and future directions. Intensive Care Med. 36, 735-49. doi: 10.1007/s00134009-1744-5.

Chang, M. I., Puder, M., \& Gura, K. M. (2012). The use of fish oil lipid emulsion in the treatment of intestinal failure associated liver disease (IFALD). Nutrients 4, 1828-50. doi: 10.3390/ nu4121828

Chen, B., Zhou, Y., Yang, P., Wan, H., \& Wu, X. (2010). Safety and efficacy of fish oil-enriched parenteral nutrition regimen on postoperative patients undergoing major abdominal surgery. J. Parenter. Enter. Nutr. 34, 387-94. doi: 10.1177/0148607110362532

Goldstein, B., Giroir, B., \& Randolph, A. (2005). International pediatric sepsis consensus conference: Definitions for sepsis and organ dysfunction in pediatrics. Pediatr. Crit. Care Med. 6, 2-8. doi: 10.1097/01. PCC.0000149131.72248.E6

Herman, R., Btaiche, I., \& Teitelbaum, D.H. (2011). Nutrition support in the pediatric surgical patient. Surg. Clin. North Am. 91, 511-41. doi: 10.1016/j.suc.2011.02.008.

Klek, S. (2016). Omega-3 fatty acids in modern parenteral nutrition: a review of the current evidence. J. Clin. Med. 5, 34. doi: 10.3390/ jem5030034.

Koletzko B., Krohn K., \& Goulet O., S.R. (2008). Pediatric parenteral nutrition : a practical reference guide. Karger, Switzerland.pp 1-66.

Koletzko, B., Goulet, O., Hunt, J., Krohn, K., \& Shamir, R. (2005). Guidelines on paediatric parenteral nutrition of the European Society of Paediatric Gastro enterology, Hepatology and Nutrition (ESPGHAN) and the European Society for Clinical Nutrition and Metabolism (ESPEN), Supported by the European Society of Paediatri. J. Pediatr. Gastroenterol. Nutr. 41, 1-87. doi: 10.1097/01.mpg.0000181841.07090. f4.

Li, N.N., Zhou, Y., Qin, X.P., Chen, Y., He, D., Feng, J.Y., \& Wu, X.T. (2013). Does intravenous fish oil benefit patients post-surgery? A meta-analysis of randomised controlled trials. Clin. Nutr. 33, 226-39. doi: 10.1016/j.clnu.2013.08.013

Manzanares, W., Langlois, P.L., Dhaliwal, R., Lemieux, M., \& Heyland, D.K. (2015). Intravenous fish oil lipid emulsions in critically ill patients: An updated systematic review and meta-analysis. Crit. Care. 19, 1-15. doi: 
10.1186/s13054-015-0888-7

Singer, P., Berger, M.M., Van den Berghe, G., Biolo, G., Calder, P., Forbes, A., Griffiths, R., Kreyman, G., Leverve, X., \& Pichard, C. (2009). ESPEN Guidelines on parenteral nutrition: intensive care. Clin. Nutr. 28, 387-400. doi: 10.1016/j.clnu.2009.04.024

The American Society for Parenteral and Enteral Nutrition (ASPEN). (2002). Guidelines for the Use of Parenteral and Enteral Nutrition in Adult and Pediatric Patients Section I : Introduction. J. Parenter. Enter. Nutr. 26, 1-138. doi: $10.1177 / 0148607102026001011$

Van Goudoever, J. B., \& Vlaardingerbroek, H., (2013). The present challenges of parenteral nutrition in preterm infants and children. Am. Soc. Nutr. 143, 2059-60. doi: 10.3945/ jn.113.176966

Vanek, V. W., Seidner, D.L., Allen, P., Bistrian, B., Collier, S., ... Kochevar, M. (2012). A.S.P.E.N. position paper: clinical role for alternative intravenous fat emulsions. Nutr. Clin. Pract. 27, 150-92. doi: 10.1177/0884533612439896

Wesson, D.E., 2018. Intestinal atresia. UpToDate. Wu, M. H., Wang, M. Y., Yang, C. Y., Kuo, M. L., \& Lin, M. T. (2014). Randomized clinical trial of new intravenous lipid (SMOFlipid 20\%) versus medium-chain triglycerides/long-chain triglycerides in adult patients undergoing gastrointestinal surgery. J. Parenter. Enter. Nutr. 38, 800-8. doi: 10.1177/0148607113512869 